\title{
Special Bertrand Curves in 4D Galilean Space
}

\author{
Handan Öztekin \\ Department of Mathematics, Firat University, 23119 Elazĭ̆, Turkey \\ Correspondence should be addressed to Handan Öztekin; handanoztekin@gmail.com
}

Received 8 October 2014; Accepted 29 November 2014; Published 22 December 2014

Academic Editor: Xiao-Qiao He

Copyright (C) 2014 Handan Öztekin. This is an open access article distributed under the Creative Commons Attribution License, which permits unrestricted use, distribution, and reproduction in any medium, provided the original work is properly cited.

\begin{abstract}
The generalization of Bertrand curves in Galilean 4-space is introduced and the characterization of the generalized Bertrand curves is obtained. Furthermore, it is proved that no special curve is a classical Bertrand curve in Galilean 4-space such that the notion of classical Bertrand curve is definite only in three-dimensional spaces.
\end{abstract}

\section{Introduction}

The geometry of curves has long captivated the interests of mathematicians, from the ancient Greeks to the era of Isaac Newton (1643-1727) and the invention of the calculus. It is a branch of geometry that deals with smooth curves in the plane and in the space by methods of differential and integral calculus. The theory of curves is simpler and narrower in scope because a regular curve in Euclidean space has no intrinsic geometry. One of the most important tools used to analyze curve is the Frenet frame, a moving frame that provides a coordinate system at each point of curve that is "best adopted" to the curve near the point.

Bertrand curves discovered by J. Bertrand in 1850 are one of the important and interesting topics of classical special curve theory. A Bertrand curve is defined as a special curve whose principal normal is the principal normal of another curve. It is characterized as curve whose curvature and torsion are in linear relation. There are many works related with Bertrand curves in the Euclidean space and Lorentzian space [1-7].

Galilean 3-space $G_{3}$ is simply defined as a Klein geometry of the product space $\mathbb{R} \times \mathbb{E}^{2}$ whose symmetry group is Galilean transformation group which has an important place in classical and modern physics. A curve in Galilean 3-space $G_{3}$ is a graph of a plane motion. Note that such a curve is called a world line in 3-dimensional Galilean space. It is well known that the idea of world lines originates in physics and was pioneered by Einstein. The term is now most often in relativity theories, that is, general relativity and special relativity.

From the differential geometric point of view, the study of curves in $G_{3}$ has its own interest. In recent years, many interesting results on curves in $G_{3}$ have been obtained by many authors (see [6-10]).

In 4-dimensional Euclidean space, generalized Bertrand curves are defined and characterized by [5]. Moreover, in 4-dimensional semi-Euclidean $E_{2}^{4}$ and also $E_{\gamma}^{n+1}(n \geq 3)$ it is proved that there is no timelike curve which is Bertrand curve [4]. Also, generalized Bertrand curves in 5-dimensional Euclidean and Lorentzian space are defined and characterized in $[6,11,12]$.

In [8], the author constructed Frenet-Serret frame of a curve in the Galilean 4-space and obtained the mentioned curve's Frenet-Serret equations.

However, to the best of our knowledge, special Bertrand curves have not been presented in the Galilean 4 -space $G_{3}$. Thus, the study is proposed to serve such a need. In this regard, we prove that there is no Frenet curve which is a classical Bertrand curve in $G_{4}$. We define and characterize $(n, e)$-Bertrand curve in four-dimensional Galilean space $G_{4}$.

\section{Preliminaries}

In this section, some fundamental properties of curves in $4 \mathrm{D}$ Galilean space are given for the purpose of the requirements [8]. 
In affine coordinates the Galilean scalar product between two points

$$
P_{i}=\left(p_{i 1}, p_{i 2}, p_{i 3}, p_{i 4}\right), \quad i=1,2,
$$

is defined by

$$
\begin{aligned}
& g\left(P_{1}, P_{2}\right) \\
& = \begin{cases}\left|p_{21}-p_{11}\right|, & \text { if } p_{21} \neq p_{11}, \\
\left(\mid\left(p_{22}-p_{12}\right)^{2}+\left(p_{23}-p_{13}\right)^{2}\right. & \\
\left.+\left(p_{24}-p_{14}\right)^{2} \mid\right)^{1 / 2}, & \text { if } p_{21}=p_{11} .\end{cases}
\end{aligned}
$$

The Galilean cross product in $G_{4}$ for the vectors $\vec{u}=$ $\left(u_{1}, u_{2}, u_{3}, u_{4}\right), \vec{v}=\left(v_{1}, v_{2}, v_{3}, v_{4}\right)$, and $\vec{w}=\left(w_{1}, w_{2}, w_{3}, w_{4}\right)$ is defined by

$$
\vec{u} \wedge \vec{v} \wedge \vec{w}=-\left|\begin{array}{cccc}
0 & e_{2} & e_{3} & e_{4} \\
u_{1} & u_{2} & u_{3} & u_{4} \\
v_{1} & v_{2} & v_{3} & v_{4} \\
w_{1} & w_{2} & w_{3} & w_{4}
\end{array}\right|
$$

where $e_{i}, 1 \leq i \leq 4$, are the standard basis vectors.

The scalar product of two vectors $\vec{U}=\left(u_{1}, u_{2}, u_{3}, u_{4}\right)$ and $\vec{V}=\left(v_{1}, v_{2}, v_{3}, v_{4}\right)$ in $G_{4}$ is defined by

$$
\langle\vec{U}, \vec{V}\rangle_{G_{4}}= \begin{cases}u_{1} v_{1}, & \text { if } u_{1} \neq 0 \text { or } v_{1} \neq 0 \\ u_{2} v_{2}+u_{3} v_{3}+u_{4} v_{4} & \text { if } u_{1}=0, v_{1}=0\end{cases}
$$

The norm of vector $\vec{U}=\left(u_{1}, u_{2}, u_{3}, u_{4}\right)$ is defined by

$$
\|\vec{U}\|_{G_{4}}=\sqrt{\left|\langle\vec{U}, \vec{U}\rangle_{G_{4}}\right|}
$$

See $[8]$.

Let $\alpha: I \subset R \rightarrow G_{4}, \alpha(s)=(s, y(s), z(s), w(s))$ be a curve parametrized by arclength $s$ in $G_{4}$. The first vector of the Frenet-Serret frame, that is, the tangent vector of $\alpha$, is defined by

$$
t=\alpha^{\prime}(s)=\left(1, y^{\prime}(s), z^{\prime}(s), w^{\prime}(s)\right) .
$$

Since $t$ is a unit vector, we can express

$$
\langle t, t\rangle_{G_{4}}=1 .
$$

Differentiating (7) with respect to $s$, we have

$$
\left\langle t^{\prime}, t\right\rangle_{G_{4}}=0
$$

The vector function $t^{\prime}$ gives us the rotation measurement of the curve $\alpha$. The real valued function

$$
\kappa(s)=\left\|t^{\prime}(s)\right\|=\sqrt{\left(y^{\prime \prime}(s)\right)^{2}+\left(z^{\prime \prime}(s)\right)^{2}+\left(w^{\prime \prime}(s)\right)^{2}}
$$

is called the first curvature of the curve $\alpha$. We assume that $\kappa(s) \neq 0$, for all $s \in I$. Similar to space $G_{3}$, the principal vector is defined by

$$
n(s)=\frac{t^{\prime}(s)}{\kappa(s)}
$$

in other words

$$
n(s)=\frac{1}{\kappa(s)}\left(0, y^{\prime \prime}(s), z^{\prime \prime}(s), w^{\prime \prime}(s)\right) .
$$

See $[8]$.

By the aid of the differentiation of the principal normal vector given in (11), define the second curvature function that is defined by

$$
\tau(s)=\left\|n^{\prime}(s)\right\|_{G_{4}} .
$$

This real valued function is called torsion of the curve $\alpha$. The third vector field, namely, binormal vector field of the curve $\alpha$, is defined by

$$
b(s)=\frac{1}{\tau(s)}\left(0,\left(\frac{y^{\prime \prime}(s)}{\kappa(s)}\right)^{\prime},\left(\frac{z^{\prime \prime}(s)}{\kappa(s)}\right)^{\prime},\left(\frac{w^{\prime \prime}(s)}{\kappa(s)}\right)^{\prime}\right) .
$$

Thus the vector $b(s)$ is perpendicular to both $t$ and $n$. The fourth unit vector is defined by

$$
e(s)=\mu t(s) \Lambda n(s) \Lambda b(s) .
$$

Here the coefficient $\mu$ is taken \pm 1 to make +1 determinant of the matrix $[t, n, b, e]$.

The third curvature of the curve $\alpha$ by the Galilean inner product is defined by

$$
\sigma=\left\langle b^{\prime}, e\right\rangle_{G_{4}} .
$$

Here, as well known, the set $\{t, n, b, e, \kappa, \tau, \sigma\}$ is called the Frenet-Serret apparatus of the curve $\alpha$. We know that the vectors $\{t, n, b, e\}$ are mutually orthogonal vectors satisfying

$$
\begin{gathered}
\langle t, t\rangle_{G_{4}}=\langle n, n\rangle_{G_{4}}=\langle b, b\rangle_{G_{4}}=\langle e, e\rangle_{G_{4}}=1, \\
\langle t, n\rangle_{G_{4}}=\langle t, b\rangle_{G_{4}}=\langle t, e\rangle_{G_{4}}=\langle n, b\rangle_{G_{4}} \\
=\langle n, e\rangle_{G_{4}}=\langle b, e\rangle_{G_{4}}=0 .
\end{gathered}
$$

For the curve $\alpha$ in $G_{4}$, we have the following Frenet-Serret equations:

$$
\begin{aligned}
& t^{\prime}=\kappa(s) n(s) \\
& n^{\prime}=\tau(s) b(s) \\
& b^{\prime}=-\tau(s) n(s)+\sigma(s) e(s), \\
& e^{\prime}=-\sigma(s) b(s) .
\end{aligned}
$$

See $[8]$.

\section{Bertrand Curves in 3-Dimensional Galilean Space}

Definition 1. Let $\alpha$ and $\bar{\alpha}$ be the curves with $\kappa_{\alpha}(s) \neq 0, \bar{\kappa}_{\alpha}(s) \neq$ $0, \tau_{\alpha}(s) \neq 0$, and $\bar{\tau}_{\alpha}(s) \neq 0$ for each $s \in I$ in $G_{3}$ and $\left\{T_{\alpha}, N_{\alpha}, B_{\alpha}\right\}$ and $\left\{\bar{T}_{\alpha}, \bar{N}_{\alpha}, \bar{B}_{\alpha}\right\}$ the Frenet frames in $G_{3}$ along $\alpha$ 
and $\bar{\alpha}$, respectively. If $\left\{N_{\alpha}, \bar{N}_{\alpha}\right\}$ is linearly dependent, in other words, if the normal lines of $\alpha$ and $\bar{\alpha}$ at $s \in I$ are parallel, then a pair of curves $(\alpha, \bar{\alpha})$ is said to be a Bertrand pair in $G_{3}$. The curve $\bar{\alpha}$ is called a Bertrand mate of $\alpha$ and vice versa. A Frenet framed curve is said to be a Bertrand curve if it admits a Bertrand mate.

Let $(\alpha, \bar{\alpha})$ be Bertrand pair in $G_{3}$. Then we can write

$$
\bar{\alpha}(s)=\alpha(s)+u(s) N_{\alpha}(s) .
$$

See [7].

Theorem 2. Let $(\alpha, \bar{\alpha})$ be Bertrand pair in $G_{3}$. Then the function $u$ defined in the above relation is a constant [7].

Theorem 3. Let $\alpha$ be a curve in $G_{3}$. Then $\alpha$ is a Bertrand curve if and only if $\alpha$ is a curve with constant torsion $\tau_{\alpha}$ [7].

Definition 4. A $C^{\infty}$-special Frenet-Serret curve $\alpha$ in 4dimensional Galilean space $G_{4}$ is called a Bertrand curve if there exist a $C^{\infty}$-special Frenet-Serret curve $\tilde{\alpha}$, distinct from $\alpha$, and a regular $C^{\infty}-\operatorname{map} \varphi: I \rightarrow \widetilde{I}(\widetilde{s}=\varphi(s), d \varphi(s) / d s \neq 0$ for all $s \in I$ ) such that curves $\alpha$ and $\tilde{\alpha}$ have the same principal normal line at each pair of corresponding points $\alpha(s)$ and $\widetilde{\alpha}(s)=\widetilde{\alpha}(\varphi(s))$ under $\varphi$. Here $s$ and $\widetilde{s}$ are arclength parameters of $\alpha$ and $\widetilde{\alpha}$, respectively. In this case, $\widetilde{\alpha}$ is called a Bertrand mate of $\alpha$ and the mate of curves $(\alpha, \widetilde{\alpha})$ is said to be a Bertrand mate in $G_{4}$.

Let $(\alpha, \widetilde{\alpha})$ be Bertrand mate in $G_{4}$. Then we can write

$$
\widetilde{\alpha}(s)=\alpha(s)+u(s) n(s) .
$$

Theorem 5. There is not any Bertrand curve in 4-dimensional Galilean space $G_{4}$.

Proof. Let $\{t, n, b, e\}$ and $\{\tilde{t}, \tilde{n}, \tilde{b}, \widetilde{e}\}$ be the Frenet-Serret frames in $G_{4}$ along $\alpha$ and $\widetilde{\alpha}$, respectively. Since $(\alpha, \widetilde{\alpha})$ is a Bertrand mate, from (19), it holds that

$$
\tilde{\alpha}(s)=\alpha(s)+u(s) n(s) .
$$

By differentiation of (20) with respect to $s$, we obtain

$$
\tilde{t}(\varphi(s)) \cdot \varphi^{\prime}(s)=t(s)+u^{\prime}(s) n(s)+u n^{\prime}(s) .
$$

From the Frenet-Serret equations, it holds that

$$
\tilde{t}(\varphi(s)) \cdot \varphi^{\prime}(s)=t(s)+u^{\prime}(s) n(s)+u \tau b .
$$

Since $\langle\widetilde{t}(\varphi(s)), \widetilde{n}(\varphi(s))\rangle=0$ and $\tilde{n}(\varphi(s))= \pm n(s)$ we get

$$
u^{\prime}(s)=0
$$

that is, $u$ is a constant function on $I$ with value $u$ (we can use the same letter without confusion). Thus (19) is rewritten as

$$
\widetilde{\alpha}(\widetilde{s})=\widetilde{\alpha}(\varphi(s))=\alpha(s)+u \cdot n(s)
$$

and we get

$$
\varphi^{\prime}(s) \tilde{t}(\varphi(s))=t(s)+u \tau(s) b(s)
$$

for all $s \in I$. By (25), we can set

$$
\tilde{t}(\varphi(s))=(\cos \theta(s)) t(s)+(\sin \theta(s)) b(s),
$$

where $\theta$ is a $C^{\infty}$-function on $I$ and

$$
\begin{aligned}
& \cos \theta(s)=\frac{1}{\varphi^{\prime}(s)}, \\
& \sin \theta(s)=\frac{u \tau(s)}{\varphi^{\prime}(s)} .
\end{aligned}
$$

Differentiating (26) and using the Frenet-Serret equations, we obtain

$$
\begin{aligned}
\varphi^{\prime}(s) & \tilde{\kappa}(\varphi(s)) \tilde{n}(\varphi(s)) \\
= & \frac{d(\cos \theta(s))}{d s} t(s) \\
& +\cos \theta(s) \kappa(s) n(s)+\frac{d(\sin \theta(s))}{d s} b(s) \\
& +\sin \theta(s)(-\tau(s) n(s)+\sigma(s) e(s)) \\
\varphi^{\prime}(s) & \widetilde{\kappa}(\varphi(s)) \tilde{n}(\varphi(s)) \\
= & \frac{d(\cos \theta(s))}{d s} t(s) \\
& +(\kappa(s) \cos \theta(s)-\tau(s) \sin \theta(s)) n(s) \\
& +\frac{d(\sin \theta(s))}{d s} b(s)+\sigma(s) \sin \theta(s) e(s) .
\end{aligned}
$$

Since $\widetilde{n}(\varphi(s))= \pm n(s)$ for all $s \in I$, we obtain

$$
\sigma(s) \sin \theta(s) \equiv 0
$$

By $\sigma(s) \neq 0$, for all $s \in I$ and (30), we get that $\sin \theta(s) \equiv 0$. Thus, by $\tau(s)>0$ and (27), we obtain that $u=0$. Therefore, (24) implies that $\tilde{\alpha}$ coincides with $\alpha$. This is a contradiction which completes the proof.

\section{Special Bertrand Curves in $G_{4}$}

In this section, we give the notion of special Bertrand curve which is called $(n, e)$-Bertrand curve in four-dimensional Galilean space $G_{4}$. We obtain a characterization of $(n, e)$ Bertrand curve.

Definition 6. Let $\alpha$ and $\widetilde{\alpha}$ be $C^{\infty}$-special Frenet-Serret curves in $G_{4}$ and $\varphi: I \rightarrow \widetilde{I}$ a regular $C^{\infty}$-map such that each point $\alpha(s)$ of $\alpha$ corresponds to the point $\widetilde{\alpha}(\widetilde{s})=\widetilde{\alpha}(\varphi(s))$ of $\widetilde{\alpha}$ for all $s \in I$. Here $s$ and $\widetilde{s}$ are arclength parameters of $\alpha$ and $\widetilde{\alpha}$, respectively. If the Frenet-Serret $\operatorname{Sp}\{n, e\}$-normal plane at each point $\alpha(s)$ of $\alpha$ coincides with the Frenet-Serret $\operatorname{Sp}\{\tilde{n}, \widetilde{e}\}$ normal plane at corresponding point $\widetilde{\alpha}(\widetilde{s})=\widetilde{\alpha}(\varphi(s))$ of $\widetilde{\alpha}$ for all $s \in I$, then $\alpha$ is called an $(n, e)$-Bertrand curve in $G_{4}$ and $\tilde{\alpha}$ is called $(n, e)$-Bertrand mate of $\alpha$. 
Theorem 7. Let $\alpha$ be $C^{\infty}$-special Frenet-Serret curves in $G_{4}$ with curvature functions $\kappa, \tau$, and $\sigma$. Then $\alpha$ is an $(n, e)-$ Bertrand curve if and only if there exist constant real numbers $\lambda, \mu, \gamma$, and $\delta$ satisfying

$$
\begin{gathered}
\lambda \tau(s)-\mu \sigma(s) \neq 0, \\
\gamma(\lambda \tau(s)-\mu \sigma(s))=1, \\
\gamma \kappa(s)-\tau(s)=\delta \sigma(s),
\end{gathered}
$$

for all $s \in I$.

Proof. We suppose that $\alpha$ is an $(n, e)$-Bertrand curve parametrized by arclength $s$. Then $(n, e)$-Bertrand mate $\widetilde{\alpha}$ is given by

$$
\widetilde{\alpha}(\widetilde{s})=\widetilde{\alpha}(\varphi(s))=\alpha(s)+\lambda(s) n(s)+\mu(s) e(s)
$$

for all $s \in I$, where $\lambda$ and $\mu$ are $C^{\infty}$-functions on $I$ and $\tilde{s}$ is the arclength parameter of $\widetilde{\alpha}$. Differentiating (31) with respect to $s$ and using the Frenet equations, we obtain

$$
\begin{aligned}
\varphi^{\prime}(s) \tilde{t}(\varphi(s))= & t(s)+\lambda^{\prime}(s) n(s) \\
& +(\lambda(s) \tau(s)-\mu(s) \sigma(s)) b(s)+\mu^{\prime}(s) e(s)
\end{aligned}
$$

for all $s \in I$.

Since the plane spanned by $n(s)$ and $e(s)$ coincides with the plane spanned by $\widetilde{n}(\varphi(s))$ and $\widetilde{e}(\varphi(s))$, we can put

$$
\begin{gathered}
\tilde{n}(\varphi(s))=\cos \theta(s) n(s)+\sin \theta(s) e(s), \\
\tilde{e}(\varphi(s))=-\sin \theta(s) n(s)+\cos \theta(s) e(s),
\end{gathered}
$$

and we notice that $\sin \theta(s) \neq 0$ for all $s \in I$. By the following facts:

$$
\begin{aligned}
0 & =\left\langle\varphi^{\prime}(s) \tilde{t}(\varphi(s)), \tilde{n}(\varphi(s))\right\rangle \\
& =\lambda^{\prime}(s) \cos \theta(s)+\mu^{\prime}(s) \sin \theta(s), \\
0 & =\left\langle\varphi^{\prime}(s) \tilde{t}(\varphi(s)), \tilde{e}(\varphi(s))\right\rangle \\
& =-\lambda^{\prime}(s) \sin \theta(s)+\mu^{\prime}(s) \cos \theta(s),
\end{aligned}
$$

we get

$$
\lambda^{\prime}(s) \equiv 0, \quad \mu^{\prime}(s) \equiv 0
$$

that is, $\lambda$ and $\mu$ are constant functions on $I$ with values $\lambda$ and $\mu$, respectively. Therefore, for all $s \in I,(31)$ is rewritten as

$$
\tilde{\alpha}(\widetilde{s})=\tilde{\alpha}(\varphi(s))=\alpha(s)+\lambda n(s)+\mu e(s)
$$

and we obtain

$$
\varphi^{\prime}(s) \tilde{t}(\varphi(s))=t(s)+(\lambda \tau(s)-\mu \sigma(s)) b(s) .
$$

Here we notice that

$$
\left(\varphi^{\prime}(s)\right)^{2}=1+(\lambda \tau(s)-\mu \sigma(s))^{2} \neq 0
$$

for all $s \in I$. Thus we can set

$$
\begin{gathered}
\tilde{t}(\varphi(s))=\cos \beta(s) t(s)+\sin \beta(s) b(s), \\
\cos \beta(s)=\frac{1}{\varphi^{\prime}(s)}, \\
\sin \beta(s)=\frac{\lambda \tau(s)-\mu \sigma(s)}{\varphi^{\prime}(s)},
\end{gathered}
$$

where $\beta$ is $C^{\infty}$-functions on $I$. Differentiating (39) with respect to $s$ and using the Frenet equations, we obtain

$$
\begin{aligned}
\varphi^{\prime}(s) & \tilde{\kappa}(\varphi(s)) \tilde{n}(\varphi(s)) \\
= & \frac{d \cos \beta(s)}{d s} t(s) \\
& +(\kappa(s) \cos \beta(s)-\tau(s) \sin \beta(s)) n(s) \\
& +\frac{d \sin \beta(s)}{d s} b(s)+\sigma(s) \sin \beta(s) e(s) .
\end{aligned}
$$

Since $\widetilde{n}(\varphi(s))$ is expressed by linear combination of $n(s)$ and $e(s)$, it holds that

$$
\frac{d \cos \beta(s)}{d s} \equiv 0, \quad \frac{d \sin \beta(s)}{d s} \equiv 0 ;
$$

that is, $\beta$ is a constant function on $I$ with value $\beta_{0}$. Thus we obtain

$$
\begin{gathered}
\tilde{t}(\varphi(s))=\cos \beta_{0} t(s)+\sin \beta_{0} b(s), \\
\varphi^{\prime}(s) \cos \beta_{0}=1, \\
\varphi^{\prime}(s) \cdot \sin \beta_{0}=\lambda \tau(s)-\mu \sigma(s)
\end{gathered}
$$

for all $s \in I$. Therefore we obtain

$$
\sin \beta_{0}=(\lambda \tau(s)-\mu \sigma(s)) \cos \beta_{0}
$$

for all $s \in I$.

If $\sin \beta_{0}=0$, then it holds that $\cos \beta_{0}= \pm 1$. Thus (43) implies that $\widetilde{t}(\varphi(s))= \pm t(s)$. Differentiating this equality, we obtain

$$
\varphi^{\prime}(s) \widetilde{\kappa}(\varphi(s)) \widetilde{n}(\varphi(s))= \pm \kappa(s) n(s)
$$

that is,

$$
\widetilde{n}(\varphi(s))= \pm n(s),
$$

for all $s \in I$. By Theorem 5, this fact is a contradiction. Thus, we must consider only the case of $\sin \beta_{0} \neq 0$. Then (45) implies

$$
\lambda \tau(s)-\mu \sigma(s) \neq 0, \quad \forall s \in I .
$$

Thus, we obtain relation (i).

The fact that $\sin \beta_{0} \neq 0$ and (46) imply

$$
(\lambda \tau(s)-\mu \sigma(s)) \frac{\cos \beta_{0}}{\sin \beta_{0}}=1
$$


and we obtain

$$
\gamma(\lambda \tau(s)-\mu \sigma(s))=1
$$

for all $s \in I$, where $\gamma=\cot \beta_{0}$ is a constant number. Thus we obtain relation (ii).

Differentiating (43) with respect to $s$ and using the Frenet equations, we obtain

$$
\begin{aligned}
\varphi^{\prime}(s) \tilde{\kappa}(\varphi(s)) \tilde{n}(\varphi(s))= & \left(\kappa(s) \cos \beta_{0}-\tau(s) \sin \beta_{0}\right) n(s) \\
& +\sigma(s) \sin \beta_{0} e(s),
\end{aligned}
$$

for all $s \in I$.

From (44) and (45) and (ii), we get

$$
\begin{aligned}
&\left(\varphi^{\prime}(s) \tilde{\kappa}(\varphi(s))\right)^{2} \\
&=\left(\varphi^{\prime}(s)\right)^{-2}\left\{[\kappa(s)-\tau(s)(\lambda \tau(s)-\mu \sigma(s))]^{2}\right. \\
&\left.+[\sigma(s)(\lambda \tau(s)-\mu \sigma(s))]^{2}\right\}
\end{aligned}
$$

for all $s \in I$.

From (38) and (51), it holds that

$$
\left(\varphi^{\prime}(s)\right)^{2}=1+\left(\frac{1}{\gamma}\right)^{2}
$$

Thus, we obtain

$$
\left[\varphi^{\prime}(s) \widetilde{\kappa}(\varphi(s))\right]^{2}=\frac{1}{\gamma^{2}+1}\left[(\gamma \kappa(s)-\tau(s))^{2}+(\sigma(s))^{2}\right] .
$$

By (44) and (45), we can set

$$
\tilde{n}(\varphi(s))=(\cos \eta(s)) n(s)+(\sin \eta(s)) e(s),
$$

where

$$
\begin{gathered}
\cos \eta(s)=\frac{\kappa(s)-\tau(s)(\lambda \tau(s)-\mu \sigma(s))}{\left(\varphi^{\prime}(s)\right)^{2} \tilde{\kappa}(\varphi(s))}, \\
\sin \eta(s)=\frac{\sigma(s)(\lambda \tau(s)-\mu \sigma(s))}{\left(\varphi^{\prime}(s)\right)^{2} \tilde{\kappa}(\varphi(s))},
\end{gathered}
$$

for all $s \in I$, where $\eta$ is $C^{\infty}$-functions on $I$.

Differentiating (56) with respect to $s$ and using the Frenet equations, we obtain

$$
\begin{aligned}
\varphi^{\prime}(s) & \tilde{\tau}(\varphi(s)) \tilde{b}(\varphi(s)) \\
= & \frac{d \cos \eta(s)}{d s} n(s)+\frac{d \sin \eta(s)}{d s} e(s) \\
& \quad+\cos \eta(s) \tau(s) b(s)-\sin \eta(s) \sigma(s) b(s),
\end{aligned}
$$

for all $s \in I$. From the above fact, it holds that

$$
\frac{d \cos \eta(s)}{d s} \equiv 0, \quad \frac{d \sin \eta(s)}{d s} \equiv 0 ;
$$

that is, $\eta$ is a constant function on $I$ with value $\eta_{0}$. Thus we obtain

$$
\begin{aligned}
& \varphi^{\prime}(s) \tilde{\tau}(\varphi(s)) \tilde{b}(\varphi(s)) \\
& \quad=\left(\cos \eta_{0} \tau(s)-\sin \eta_{0} \sigma(s)\right) b(s) .
\end{aligned}
$$

Since $\varphi^{\prime}(s) \widetilde{\tau}(\varphi(s)) \widetilde{b}(\varphi(s)) \neq 0$ for all $s \in I$, it holds that

$$
\cos \eta_{0} \tau(s)-\sin \eta_{0} \sigma(s) \neq 0
$$

Let $\delta=\cos \eta_{0} / \sin \eta_{0}$ be a constant number. Then (51) and (57) imply

$$
\begin{gathered}
\delta=\frac{\kappa(s)-\tau(s)(\lambda \tau(s)-\mu \sigma(s))}{\sigma(s)(\lambda \tau(s)-\mu \sigma(s))}, \\
\gamma \kappa(s)-\tau(s)=\delta \sigma(s) ;
\end{gathered}
$$

that is, we obtain relation (iii).

Conversely, we suppose that $\alpha$ is a $C^{\infty}$-special Frenet curve in $G_{4}$ with curvature functions $\kappa, \tau$, and $\sigma$ satisfying (i), (ii), and (iii) for constant numbers $\lambda, \mu, \gamma$, and $\delta$. Then we define a $C^{\infty}$-curve $\widetilde{\alpha}$ by

$$
\widetilde{\alpha}(s)=\alpha(s)+\lambda n(s)+\mu e(s)
$$

for all $s \in I$, where $s$ is the arclength parameter of $\alpha$. Differentiating (63) with respect to $s$ and using the Frenet equations, we obtain

$$
\frac{d \widetilde{\alpha}(s)}{d s}=t(s)+(\lambda \tau(s)-\mu \sigma(s)) b(s)
$$

for all $s \in I$. Thus, by relation (ii), we obtain

$$
\frac{d \widetilde{\alpha}(s)}{d s}=t(s)+\frac{1}{\gamma} b(s) .
$$

Since relation (i) holds, the curve $\widetilde{\alpha}$ is a regular curve. Then there exists a regular map $\varphi: I \rightarrow \widetilde{I}$ defined by

$$
\widetilde{s}=\varphi(s)=\int_{0}^{s}\left\|\frac{d \widetilde{\alpha}(t)}{d t}\right\| d t, \quad(\forall s \in I),
$$

where $\widetilde{s}$ denotes the arclength parameter of $\widetilde{\alpha}$, and we obtain

$$
\varphi^{\prime}(s)=\varepsilon \sqrt{1+(\lambda \tau(s)-\mu \sigma(s))^{2}}=\varepsilon \sqrt{1+\frac{1}{\gamma^{2}}}, \quad \varepsilon=\mp 1,
$$

for all $s \in I$. Thus the curve $\widetilde{\alpha}$ is rewritten as

$$
\widetilde{\alpha}(\widetilde{s})=\widetilde{\alpha}(\varphi(s))=\alpha(s)+\lambda n(s)+\mu e(s)
$$

for all $s \in I$. Differentiating the above equality with respect to $s$, we obtain

$$
\left.\varphi^{\prime}(s) \frac{d \widetilde{\alpha}(\widetilde{s})}{d \widetilde{s}}\right|_{\widetilde{s}=\varphi(s)}=t(s)+\frac{1}{\gamma} b(s) .
$$


We can define a unit vector field $\widetilde{t}$ along $\widetilde{\alpha}$ by $\widetilde{t}(\widetilde{s})=$ $d \widetilde{\alpha}(\widetilde{s}) / d \widetilde{s}$ for all $\widetilde{s} \in \widetilde{I}$. By (67) and (69) we obtain

$$
\tilde{t}(\widetilde{s})=\varepsilon\left(1+\frac{1}{\gamma^{2}}\right)^{-1 / 2} \cdot\left(t(s)+\frac{1}{\gamma} b(s)\right)
$$

for all $s \in I$. Differentiating (70) with respect to $s$ and using the Frenet equations, we obtain

$$
\begin{aligned}
& \left.\varphi^{\prime}(s) \frac{d \widetilde{t}(\widetilde{s})}{d \widetilde{s}}\right|_{\tilde{s}=\varphi(s)} \\
& =\varepsilon\left(1+\frac{1}{\gamma^{2}}\right)^{-1 / 2} \cdot\left(\kappa(s)-\frac{\tau(s)}{\gamma}\right) n(s)+\frac{\sigma(s)}{\gamma} e(s), \\
& \left\|\left.\frac{d \widetilde{t}(\widetilde{s})}{d \widetilde{s}}\right|_{\tilde{s}=\varphi(s)}\right\|=\frac{\sqrt{(\kappa(s)-(\tau(s) / \gamma))^{2}+(\sigma(s) / \gamma)^{2}}}{\varphi^{\prime}(s) \sqrt{1+\left(1 / \gamma^{2}\right)}} .
\end{aligned}
$$

By the fact that $\varphi^{\prime}(s)>0$ for all $s \in I$, we obtain

$$
\widetilde{\mathcal{\kappa}}(\varphi(s))=\left\|\left.\frac{d \widetilde{t}(\widetilde{s})}{d \widetilde{s}}\right|_{\tilde{s}=\varphi(s)}\right\|>0
$$

for all $s \in I$. Then we can define a unit vector field $\widetilde{n}$ along $\widetilde{\alpha}$ by

$$
\begin{aligned}
\tilde{n}(\tilde{s})= & \tilde{n}(\varphi(s)) \\
= & \frac{1}{\varepsilon \sqrt{(\kappa(s)-(\tau(s) / \gamma))^{2}+(\sigma(s) / \gamma)^{2}}} \\
& \times\left(\left(\kappa(s)-\frac{\tau(s)}{\gamma}\right) n(s)+\frac{\sigma(s)}{\gamma} e(s)\right)
\end{aligned}
$$

for all $s \in I$. Thus we can put

$$
\widetilde{n}(\varphi(s))=\cos \xi(s) \cdot n(s)+\sin \xi(s) \cdot e(s),
$$

where

$$
\begin{aligned}
& \cos \xi(s)=\frac{\gamma \kappa(s)-\tau(s)}{\varepsilon \gamma \sqrt{(\kappa(s)-(\tau(s) / \gamma))^{2}+(\sigma(s) / \gamma)^{2}}} \\
& \sin \xi(s)=\frac{\sigma}{\varepsilon \gamma \sqrt{(\kappa(s)-(\tau(s) / \gamma))^{2}+(\sigma(s) / \gamma)^{2}}}
\end{aligned}
$$

for all $s \in I$. Here $\xi$ is a $C^{\infty}$-function on I. Differentiating (74) with respect to $s$ and using the Frenet equations, we get

$$
\begin{aligned}
\varphi^{\prime}(s) & \left.\cdot \frac{d \widetilde{n}(\widetilde{s})}{d \widetilde{s}}\right|_{\tilde{s}=\varphi(s)} \\
= & \cos \xi(s) \cdot \tau(s) b(s)+\frac{d \cos \xi(s)}{d s} n(s) \\
& \quad+\sin \xi(s) \cdot(-\sigma(s) b(s))+\frac{d \sin \xi(s)}{d s} e(s) .
\end{aligned}
$$

Differentiating (iii) with respect to $s$, we obtain

$$
\gamma \kappa^{\prime}(s)-\tau^{\prime}(s)-\delta \sigma^{\prime}(s) \equiv 0 .
$$

Differentiating (74) and (75) with respect to $s$ and using (78), we get

$$
\frac{d \cos \xi(s)}{d s} \equiv 0, \quad \frac{d \sin \xi(s)}{d s} \equiv 0 ;
$$

that is, $\xi$ is a constant function on $I$ with value $\xi_{0,}$. Thus, we find

$$
\begin{aligned}
& \cos \xi_{0}=\frac{\gamma \kappa(s)-\tau(s)}{\varepsilon \sqrt{(\gamma \kappa(s)-\tau(s))^{2}+(\sigma(s))^{2}}}, \\
& \sin \xi_{0}=\frac{\sigma(s)}{\varepsilon \sqrt{(\gamma \kappa(s)-\tau(s))^{2}+(\sigma(s))^{2}}} .
\end{aligned}
$$

From (74), it holds that

$$
\widetilde{n}(\varphi(s))=\cos \xi_{0} n(s)+\sin \xi_{0} e(s) .
$$

Thus we obtain, by (70) and (72),

$$
\begin{aligned}
\widetilde{\kappa}(\varphi(s)) \widetilde{t}(\varphi(s))= & \frac{\sqrt{(\kappa(s)-(\tau(s) / \gamma))^{2}+(\sigma(s) / \gamma)^{2}}}{\varepsilon \varphi^{\prime}(s)(1+(1 / \gamma))^{2}} \\
& \times\left(t(s)+\frac{1}{\gamma} b(s)\right) .
\end{aligned}
$$

Thus we can define a unit vector field $\widetilde{b}(\widetilde{s})$ along $\widetilde{\alpha}$ by

$$
\widetilde{b}(\varphi(s))=\varepsilon\left(1+\frac{1}{\gamma^{2}}\right)^{-1 / 2}\left(-\frac{1}{\gamma} t(s)+b(s)\right)
$$

for all $s \in I$.

Next we can define a unit vector field $\widetilde{e}(\widetilde{s})$ along $\widetilde{\alpha}$ by

$$
\widetilde{e}(\widetilde{s})=\widetilde{e}(\varphi(s))=-\sin \xi_{0} n(s)+\cos \xi_{0} e(s)
$$

for all $s \in I$. Now we obtain, by (70), (74), (81), and (83),

$$
\begin{gathered}
\operatorname{det}[\widetilde{t}(\varphi(s)), \widetilde{n}(\varphi(s)), \widetilde{b}(\varphi(s)), \widetilde{e}(\varphi(s))] \\
=\operatorname{det}[t(s), n(s), b(s), e(s)]=0, \\
\langle\widetilde{t}, \widetilde{n}\rangle=\langle\widetilde{t}, \widetilde{b}\rangle=\langle\widetilde{t}, \widetilde{e}\rangle=\langle\widetilde{n}, \widetilde{e}\rangle \\
=\langle\widetilde{n}, \widetilde{b}\rangle=\langle\widetilde{b}, \widetilde{e}\rangle=0, \\
\langle\widetilde{t}, \tilde{t}\rangle=\langle\widetilde{n}, \widetilde{n}\rangle=\langle\widetilde{b}, \widetilde{b}\rangle=\langle\widetilde{e}, \widetilde{e}\rangle=1,
\end{gathered}
$$

for all $s \in I$. Thus the frame $\{\widetilde{t}, \widetilde{n}, \widetilde{b}, \widetilde{e}\}$ along $\widetilde{\alpha}$ is orthonormal and positive. Therefore the curve $\widetilde{\alpha}$ is a $C^{\infty}$-special FrenetSerret curve in $G_{4}$, and it is obvious that the Frenet-Serret $\operatorname{Sp}\{n, e\}$-normal plane at each point $\alpha(s)$ of $\alpha$ coincides with the Frenet-Serret $\operatorname{Sp}\{\tilde{n}, \tilde{e}\}$-normal plane at corresponding point $\widetilde{\alpha}(\widetilde{s})$ of $\widetilde{\alpha}$. Therefore $\alpha$ is an $(n, e)$-Bertrand curve in $G_{4}$. 


\section{Conflict of Interests}

The author declares that there is no conflict of interests regarding the publication of this paper.

\section{References}

[1] H. Balgetir, M. Bektas, and M. Ergüt, "Bertrand curves for nonnull curves in 3-dimensional Lorentzian space," Hadronic Journal, vol. 27, no. 2, pp. 229-236, 2004.

[2] J. H. Choi, T. H. Kang, and Y. H. Kim, "Bertrand curves in 3dimensional space forms," Applied Mathematics and Computation, vol. 219, no. 3, pp. 1040-1046, 2012.

[3] M. P. Do Carmo, Differential Geometry of Curves and Surfaces, Pearson Education, 1976.

[4] N. Ekmekci and K. Ilarslan, "On Bertrand curves and their characterization," Differential Geometry-Dynamical Systems, vol. 3, no. 2, pp. 17-24, 2001.

[5] H. Matsuda and S. Yorozu, "Notes on Bertrand curves," Yokohama Mathematical Journal, vol. 50, no. 1-2, pp. 41-58, 2003.

[6] Z. Nadenik, "Bertrand curves in five-dimensional space," Czechoslovak Mathematical Journal, vol. 2, no. 1, pp. 57-87, 1952 (Russian).

[7] A. O. Öğrenmiş, H. Öztekin, and M. Ergüt, "Bertrand curves in Galilean space and their characterizations," Kragujevac Journal of Mathematics, vol. 32, pp. 139-147, 2009.

[8] S. Yilmaz, "Construction of the Frenet-Serret frame of a curve in 4D Galilean space and some applications," International Journal of Physical Sciences, vol. 5, no. 8, pp. 1284-1289, 2010.

[9] H. Öztekin and A. O. Öğrenmiş, "Normal and rectifying curves in pseudo-Galilean space $G(1 / 3)$ and their characterizations," Journal of Mathematical and Computational Science, vol. 2, no. 1, pp. 91-100, 2012.

[10] A. O. Ogrenmis, M. Ergut, and M. Bektas, "On the helices in the Galilean space $G_{3}$," Iranian Journal of Science and Technology Transaction A: Science, vol. 31, no. 2, pp. 177-181, 2007.

[11] S. Ersoy and A. Inalcik, "Generalized spacelike Bertrand curves in Minkowski 5-space $\mathbb{R}_{1}^{5}$," Quaestiones Mathematicae, vol. 37, no. 1, pp. 19-29, 2014.

[12] B. Divjak and Š. Ž. Milin, "Special curves on ruled surfaces in GALilean and pseudo-GALilean spaces," Acta Mathematica Hungarica, vol. 98, no. 3, pp. 203-215, 2003. 


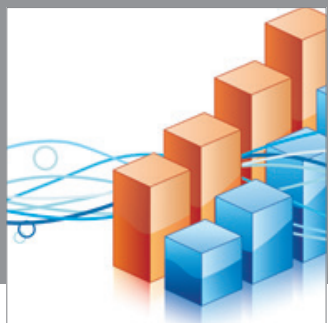

Advances in

Operations Research

mansans

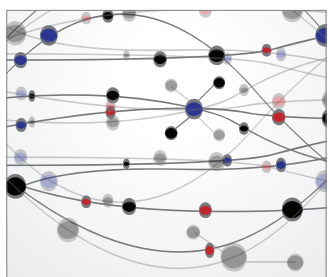

The Scientific World Journal
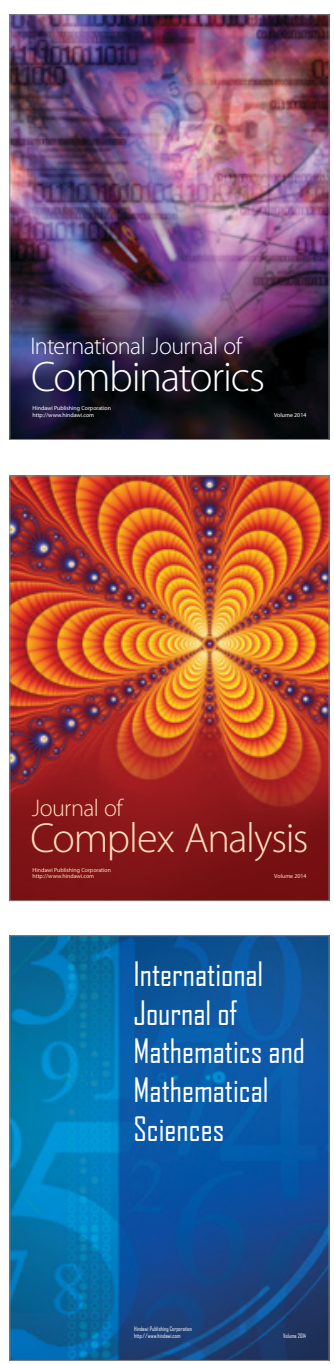
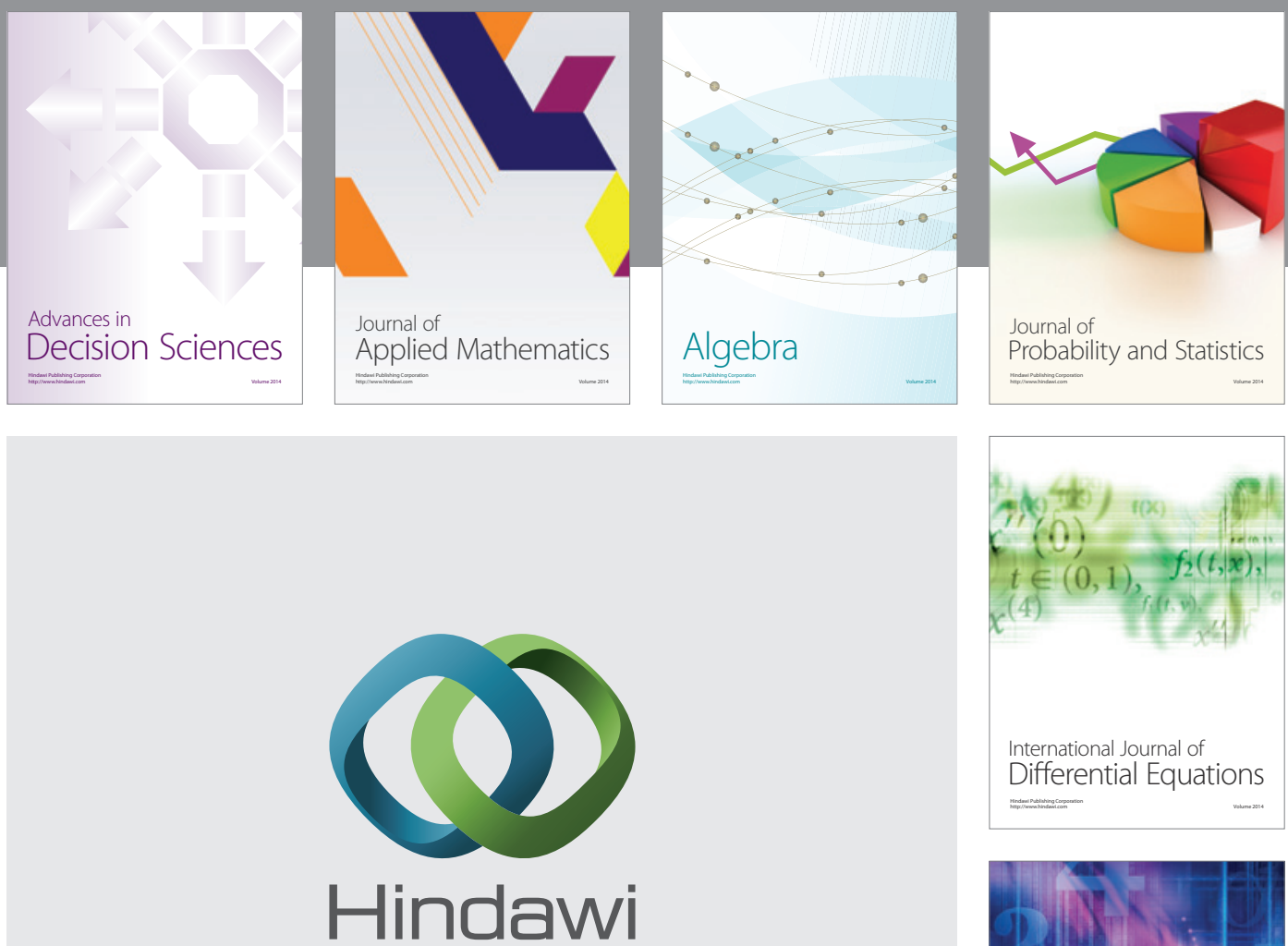

Submit your manuscripts at http://www.hindawi.com
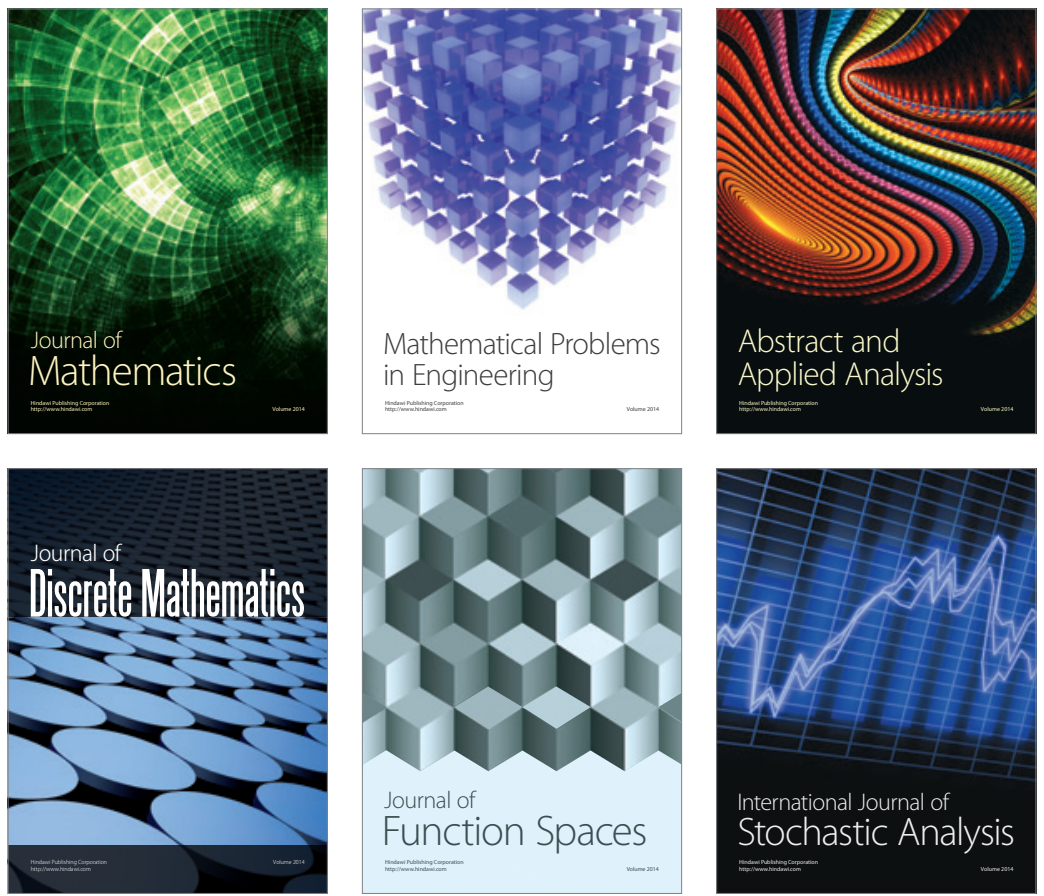

Journal of

Function Spaces

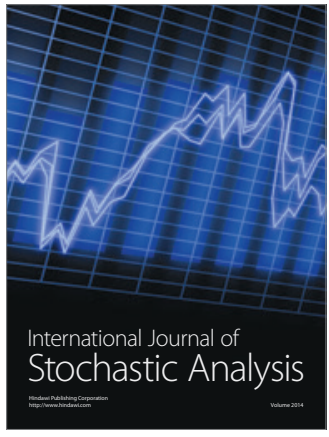

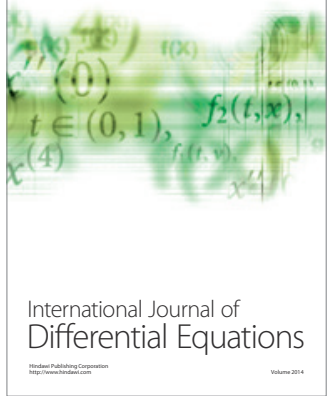
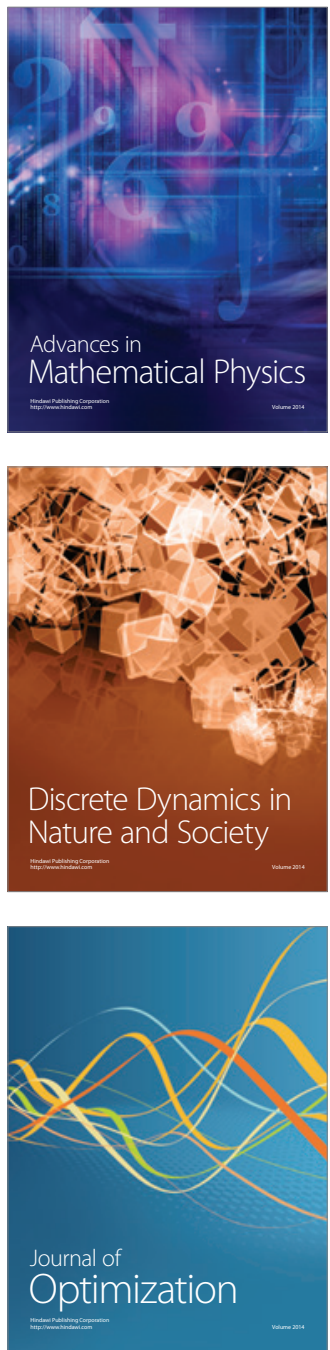